\title{
Building Dependable EEG Classifiers for the Real World - It's Not Just about the Hardware
}

\author{
Gene Davis ${ }^{1}$, Djordje Popovic ${ }^{1,2}$, Robin R. Johnson ${ }^{1}$, Chris Berka ${ }^{1}$, \\ and Mirko Mitrovic ${ }^{1}$ \\ ${ }^{1}$ Advanced Brain Monitoring, Inc. 2237 Faraday Avenue, Suite 100, Carlsbad, CA 92008 \\ ${ }^{2}$ University of Southern California, Los Angeles, CA 90089 \\ \{Gene, Dpopovic, Rjohnson, Chris, Mirkom\} ab-alert.com
}

\begin{abstract}
One of the major deficiencies with the EEG-based classifiers used in today's laboratory settings is that they are often ill suited for the real world. In many cases the classifiers that were painstakingly developed in the controlled laboratory environment become unreliable with increased mobility of the user. In addition to increased mobility, many real world scenarios impose constraints on data collection that cannot be accommodated by the lab-created classifier. Addressing these issues throughout the development process of EEG-based classifiers by building hardware, software, and algorithms intended for use in the real world should result in more dependable classifiers. With this approach we were able to collect and classify data on a research vessel at sea, in the desert by night, on dismounted soldiers in the training field, and everywhere between.
\end{abstract}

Keywords: Electroencephalogram (EEG), Mobile EEG, Operational Neuroscience, Engagement, Workload, Drowsiness.

\section{Introduction}

Researchers have been interested in developing electroencephalogram (EEG) based classifiers for over forty years, in order to enable brain-computer interface (BCI) and neuro-feedback applications. Classifiers have been developed to track global activation state(s) as well as specific cognitive and medically diagnostic states [1-5]. Many commercially available EEG systems allow for the simultaneous recording of high quality EEG from a large number of scalp locations $(128-256)$ in controlled laboratory environments and the advent of powerful digital electronics has allowed a shift in focus from simple data collection to development and implementation of complex signal processing and pattern recognition techniques that can be programmed to run in real-time. EEG metrics have been developed to quantify alertness, engagement, drowsiness and working memory, and the integration of EEG metrics into the evaluation of Human-Computer Interfaces provided the foundation for establishing new fields including neuroergonomics [1, 6-14]. However, the use of EEG outside of the lab was, until recently hampered by high susceptibility of EEG to movement artifacts, as well as environmental and physiological noise with amplitudes several orders of magnitude larger than the typical EEG amplitude $(20-50 \mu \mathrm{V})$. Only in the last decade 
has EEG left the laboratory, and entered the field with the development of portable EEG systems that include the acquisition tools and noise rejection techniques necessary for high quality data collection in real world environments. Having these tools and systems enables implementation of EEG-based classifiers in real time in the real world.

Real-world, real-time EEG applications must meet certain requirements to be relevant and useful. First, the equipment, software and algorithms required for acquisition and processing must be easy to set-up and use. The hardware needs to be mobile, lightweight, robust, flexible, and reliable, whereas the acquisition and processing software should be intuitive and flexible. Finally, the algorithms should be developed with real-world applications in mind. While there are a number of potential approaches to developing real-world, real-time classifiers, at a minimum the hardware, software, and algorithm development methodology must be part of the overall game plan.

\section{Methods}

\subsection{It's All about the Hardware}

Hardware for in-field acquisition of EEG must address the following issues: portability, ease of set-up and use in the field, durability, and minimization of signal acquisition artifacts. Mobility and portability requirements translate into a need for lightweight, simplified form factors, and preferably a wireless system. Wireless systems provide maximal flexibility and platform-independence in addition to improving durability while reducing environmental artifact during acquisition. To meet ease of use requirements, it should be easy to apply by the end user and once applied, it should become "transparent" so the user is able to focus on the task at hand (not the equipment). Finally the system should be easy to trouble shoot with minimal set up time.

Advanced Brain Monitoring (ABM) has developed a system that meets these requirements. The physical system consists of 3 basic components plus electrodes: a skull cap, and electrode placement strip that is integrated into the skull cap, and an electronics headset. The skull cap and electrode placement strip components are sizematched, and sizing is derived from the Nasium-to-Occipital distance of the end user, allowing selection of small, medium or large sizes. The integrated, size-matched skull cap and electrode placement strip incorporates the occipital bone placement to ensure accurate and easy placement of the electrodes (according to the 10-20 system). This set up has the added advantage of allowing easy identification and correction of incorrect sizing that could lead to inappropriate placement of the electrodes and collection of faulty data. The number of electrodes used is also important in minimizing the setup time, maintaining a light weight system, and reducing power consumption requirements that can decrease portability, durability, and flexibility. With only three electrodes, (Fz, Cz, and POz) the ABM system classifies a subject's arousal state on a spectrum from Sleep Onset to High Engagement [6, 15-25]. By adding only three additional electrodes, (F3, C3, and C4), cognitive workload states have been determined in multiple Augmented Cognition scenarios [17, 19, 26 - 30]. Finally, impedances below $5 \mathrm{kOhms}$ are not essential, and therefore need not delay the start of data 
collection. With the current ABM set-up data can be collected and classified with impedances as high as $45 \mathrm{kOhms}$, allowing for much greater flexibility in real-world environments. All of the above allow the system to be set-up and begin data collection quickly in the field setting, and are particularly advantageous in time sensitive settings where access to the subject population may be limited or driven by inflexible third party events.

The system used for the majority of data collection to date is a Bluetooth (BT) wireless system that allows for true mobility in any setting. Wireless EEG has been acquired and passed to PDA's, desktops, laptops, and mini-laptops; allowing the user to move freely in any environment without fear of catching loose wires, while providing maximal flexibility in the set-up process. Additional mobility and comfort is found in the design of the EEG cap. Unlike traditional EEG caps, that use a chin strap to hold the EEG cap in place, the ABM system uses a skull cap design that holds the cap in place by applying tension around the head (similar to a head band) providing a greater range of normal movement. The end result is hardware that weighs less than 6 $\mathrm{oz}$ including the electrodes, cap, electronics and batteries in a system with maximal run times of over 10 hours on two AAA batteries.

The hardware discussed herein, has a small vertical and horizontal footprint that allows the system to be integrated into multiple configurations on the end-user. The condensed size has allowed the system to be integrated with and under, Kevlar and safety helmets, fNIR headsets, gas masks and various head mounted eye trackers [31-33]. The system design provided the flexibility that accommodated these various configurations: the flexible cap and strip material and configuration provided the robustness needed to maintain good scalp contact required for high quality data acquisition with minimal noise in the signal. The electronics that provide amplification and digitization, as well as BT transmission are encased in a plastic reinforced with Lexan material, for high impact resistance. The durability and robustness of the hardware system was perhaps best demonstrated during use in live-fire, Simunitions training exercises occurring in a rainstorm at Aberdeen proving grounds [34], where the headset sustained a direct hit, yet maintained full functionality, continuing to acquire high quality data. In the real world, a system that acquires data for a classifier will need to be ready for anything, including: rain, misuse, abuse, and neglect- and yet be able to continue to collect high quality EEG signals that are the basis of the classifier.

Ideally, the hardware design should minimally impact the state of the user; they should forget that the system is on. Depending on the level of intrusiveness of the hardware, the classifications characterizing distraction, work load, or effort described may be from the task, from coping with the data collection system, or any combination of related factors. By incorporating comfort into the original design of the ABM system the end result is a system that has been worn for 24 hours of continuous use, and has been worn by over 1000 subjects with minimal awareness of the data collection system.

\subsection{It's All about the Software}

Real-world software for acquisition of EEG must address the following issues: At minimum, the software required for acquisition and processing must be easy to install and intuitive to use. Ideally it should also provide mobility options, along with 
flexible interfaces, inputs and outputs, and reliably collect and save data. The software would be of greater assistance to the non-EEG researcher as well as the experienced EEG researcher if it were also able to identify and provide feedback regarding artifacts associated with environmental noise and/or physiological noise. Finally, the software should allow for flexible interaction with third-party software programs to ensure maximal applications of the classifiers developed.

The B-Alert Acquisition software developed by ABM was designed to meet the above needs. The software for running data acquisition in the field is less than 50 megabytes in size, can be installed in under five minutes, and does not require any additional third party software or drivers making it truly plug and play. This platform allows acquisition through a desktop, laptop, mini-computer, or PDA. As computer issues are not uncommon in real-world applications, this system allows for quick adaptation to another collection interface to be adapted as needed (either through changing to another similar system such as an additional laptop; or changing systems completely as needed such as from a laptop to a PDA). With this high degree of simplicity and flexibility, researchers are enabled to develop a plethora of experimental designs and applications.

The software has simple selection options for acquisition and retransmission allowing for observation of the signal quality from a secondary computer. In addition, prior to acquisition the software completes both an impedance check and an artifact evaluation for each electrode. Thus, the researcher can have great confidence in the initial signal quality. Finally, while in Acquisition mode(s) the software provides helpful feedback (regarding artifact as well as overall signal quality) to the end user allowing even an untrained EEG technician to quickly recognize and troubleshoot poor EEG signal quality.

The ABM B-Alert Acquisition software suite has been used to provide input from EEG into several other third party systems to enable closed-loop feedback systems. The algorithms for identifying arousal and workload states may be passed (as would any additional algorithms that may developed), as well as the raw EEG signal. These options are easily enabled, and can be used to develop and apply real-time feedback systems in field operational environments. The configurations enabled in the B-alert acquisition software allow researchers to collect the developmental data for realworld algorithms in the real world. Perhaps equally important, the system also then enables application of these algorithms (once developed and validated) in the real world. In several recent collaborations with the Lockheed-Martin Advanced Technology Laboratory, the ABM software provided outputs via DLL (digital library link) on operator levels of engagement and mental workload that were used to drive changes in the display of a Tactical Tomahawks Weapons training simulation. The resulting closed-loop system provided an $80 \%$ reduction in launch time deviations from optimal and a 55\% reduction in the number of late launches [26].

\subsection{Its All about the Algorithms}

Real world algorithms must be flexible, robust, and developed with real-world applications in mind. To meet these requirements, the following issues will need to be considered: the design of the initial data collection experiments upon which the classifiers will be built, the feature extraction method for model building, the types of classifier 
models that may be considered, and the validation of the classifier. Ideally, the initial experimental design will be informed by the planned methodology for mathematically building the classifier, and these will both inform the validation process. In other words, the development of a classifier should be a well-designed rational process based on a plan that takes into account the final application(s) envisioned for the classifier.

Experimental design for initial data collection will need to address many aspects of the planned research. First, the overall design of the experiment should be considered, to ensure proper experimental control of unexplainable variance that may de-stabilize the models that are developed. Full factorial designs are not required, but well thought out protocols will ensure that the appropriate data (such as ERPs) are in fact available for classifier development. It is also essential that all subjects are exposed to identical scenarios for the purposes of development- casual changes will lead to noise that will negatively impact algorithm development. The experimental design for the initial data collection should include an acknowledgement of real-world concerns: and may benefit greatly from at least some real-world data.

Next, understanding the number and quality of anticipated features to be extracted must be taken into consideration in regard to the sample size selected. In general, stable mathematical models with good generalization ability require a minimum sample size that is at least one order of magnitude larger than the number of features extracted per unit of observation (e.g. a single-trial ERP waveform) and subsequently used for classification. Classifiers that are built on a sample of insufficient size tend to memorize rather than learn from the sample, which usually results in poor 'real world' performance on cross-validation in spite of high classification accuracy achieved during the model development. This problem is aggravated by complexity of the selected classifier (e.g. non-linear classifiers are more vulnerable than linear, neural networks with more hidden layer neurons are more easily over-trained than simpler architectures) or by non-linear transformations of the input space, typical for support vector machines (SVM), that in a non-transparent way significantly increase the real number of features (often as $\sim \mathrm{O}\left(\mathrm{N}^{2}\right)$ or $\sim \mathrm{O}\left(\mathrm{N}^{3}\right)$, where $\mathrm{N}$ is the number of 'visible', i.e. nominal features in the original input space). Other issues may also impact the sample size that can be reasonably collected, including access to the appropriate subjects.

Building a classifier (in a broad sense of the word) can take a number of paths, but it usually consists of three tightly connected phases: choice of feature extraction method(s), choice of feature selection method(s) and finally selection of classifier in a narrower sense of the word. Feature extraction methods serve to reduce the dimensionality of the original space containing input variables by determining an appropriate subspace of, in most cases, smaller dimensionality. Principal component analysis (PCA) is the most popular technique for dimensionality reduction, but other linear transforms (factor analysis, linear discriminant analysis, projection pursuit) or nonlinear techniques (Kernel PCA, multi-dimensional scaling, and self-organizing maps) can be used instead. One should note that for low-dimensionality input spaces feature extraction can be omitted. Feature selection methods take the original or transformed set of $\mathrm{N}$ input variables and down-selects a subset of $\mathrm{M}(\mathrm{M}<\mathrm{N})$ variables that provide the best discrimination among the different classes into which signals (e.g. an ERP) should be classified. Exhaustive search, branch-and-bound search, sequential forward 
or backward search, plus-L-take-away-R selection and sequential forward and backward floating search are some of the popular methods available to researchers. The key for a successful feature selection is the balance between the optimality of a method (i.e. how likely it is to find a local rather than the global minimum) and the speed of convergence (e.g. branch-and-bound search is optimal but very slow). Finally, a variety of techniques is available that will group similar vectors of selected features into few desired classes such as the k-nearest neighbor rule, binary decision tree, Bayes classifier, logistic classifier, Parzen classifier, Fisher linear discriminants, support vector machines and neural networks. Because of the existence of so many (in some respects overlapping) techniques and ready availability of fast computers nowadays, attempts at building a classifier easily turn into a fishing expedition. Ideally, the researcher will understand the goal of the classifier and select the appropriate techniques in order to arrive at a solid solution.

Finally, validation of any classifier must be completed prior to finalization and application. Validation may be done on the initial data set using various techniques, including bootstrapping, and hold out methods. While such an approach is statistically legitimate and can save both time and financial resources, it may not be sufficient to ensure true real-world generalized applicability. Therefore, it is highly desirable to ensure that once developed, the classifier be cross-validated on a unique dataset different from that used for the development of the classifier. Over time, occasional reexamination of the classifier's performance as it is used can also serve to ensure that it maintains real-world viability.

\section{Results}

Classifiers that have been successfully collected include both arousal state (sleep onset-distraction-low engagement-high engagement) and cognitive workload (low-high). Over 1000 subjects have had data collected in over 2000 sessions by over 30 in-house technicians at ABM, and our client/collaborators have collected hundreds of additional subjects with over 50 client/collaborator technicians. Through ABM work and that of our collaborators, EEG data has been collected on soldiers in the field at 29 Palms, in live-fire exercises at Aberdeen Training Grounds inNorfolk, Virginia, and Camp Pendleton (San Diego, CA), in vehicles, and in the classroom. EEG data has been collected from expert imagery analysts, expert marksman, expert chemistry
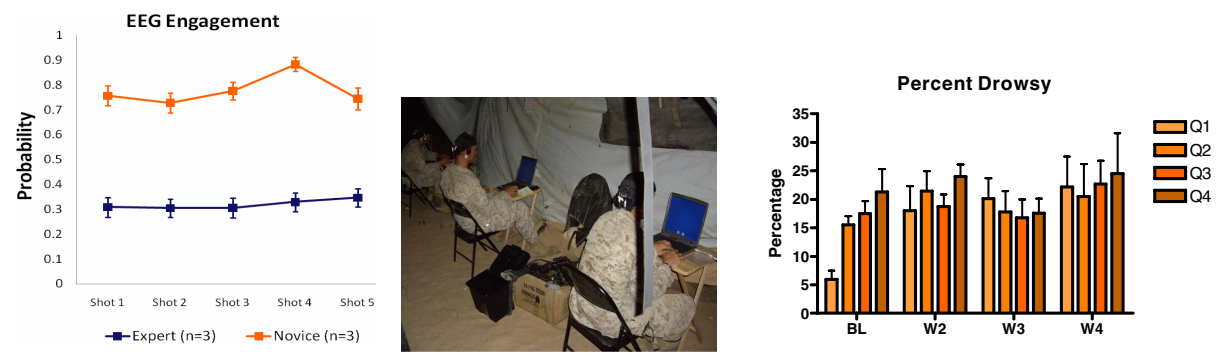

Fig. 1. From left to right: Engagement levels during marksmanship studies, data collection at 29 Palms, drowsiness classifications for 29 Palms study 
students, as well as a plethora of healthy control volunteers, novice marksman, sleep disordered patients, and other experimental subjects. Some exemplar data results are shown in Figure 1.

\section{Conclusion}

Investigations of human mental activity have employed EEG recordings for nearly a century since the first recordings were made by Hans Berger in 1929 [35]. Today EEG is routinely used for overnight sleep studies in the laboratory and in neurology to characterize epilepsy and neurological disorders, but the great leaps in EEG research can be largely attributed to the wealth of information generated by psychologists and neuroscientists using EEG to investigate brain, mind and behavior. Although the relationships between specific mental states and EEG are just beginning to be understood, the foundation of work in detecting global state changes is sufficient to begin developing practical applications. Our team developed hardware and software to facilitate the widespread and routine use of EEG outside the laboratory supporting a growing number of applications in education and training, human factors evaluations, military operations and market research. Our team developed methods for Psychophysiological Profiling that can now be accomplished by integrating EEG and EKG with cognitive tests. The result is efficient, inexpensive assessment of alertness, attention, learning and memory, providing a quantitative profile of impairment that can be used for patients with sleep, neurological and psychiatric disorders. These NeuroAssays can be used for diagnostic and treatment outcome evaluations, pharmaceutical investigations, and to identify potential biomarkers for specific diseases [15-17].

Field applications for the EEG technology include real-time assessment of drowsiness for truck drivers or airline pilots in military, industrial or other operational settings. The integration of EEG monitoring of operator status offers the possibility of allocating tasks between machines and humans based on the operator status. Intelligent feedback or "closed-loop" systems can facilitate active intervention by the operator or through a third party (man or machine), increasing safety and productivity. Another novel approach to this evolving technology is to radically rethink the design of human-machine system interfaces to optimize the flow and exchange of data between humans and machines. The new discipline of neuroergonomics has taken on this challenge combining understanding of the neural bases of cognition and behavior with the design and implementation of technology. Our team is collaborating with educators in developing next generation EEG technology to build models of student learning using EEG for non-intrusive assessment of cognitive processes including attention, working memory, workload and problem solving [29-30]. The vision is to integrate EEG into interactive tutorials and training simulations. There are no limits to the applications of the venerable technology now that it has left the laboratory.

Although the hardware, software, and algorithms are continually undergoing changes to provide better end results, the current complete package to date has been used in a variety of locations, weather conditions, and configurations that were previously impractical if not impossible to collect EEG data in with other commercially 
available systems. Our system will continue to be developed to improve the durability, flexibility, and applicability in the real world, including the development of classifiers for the real world.

Acknowledgements. This work was supported by NIHINIMH Grant No. MH078436, NIHINHLBI Grant No. HL70484, NIHINIMH Grant No. MH64344-01, DARPAIDSO Grant No. 20064169, DARPA Accelerated Learning Program, Dr. Amy Kruse, PM and theDARPA Augmented Cognition program, Drs. Dylan Schmorrow \& Amy Kruse, PM.

"A" (Approved for Public Release, Distribution Unlimited).

\section{References}

1. Besserve, M., Jerbi, K., Laurent, F., et al.: Classification methods for ongoing EEG and MEG signals. Biological Research 40, 415-437 (2007)

2. Gevins, A.S., Morgan, N.H.: Classifier-directed signal processing in brain research. IEEE Transactions on Biomedical Engineering 33, 1054-1068 (1986)

3. Mirsky, A.F., Pragay, E.B.: The relation of EEG and performance in altered states of consciousness, vol. 45, pp. 514-534. Research Publications - Association for Research in Nervous and Mental Disease (1967)

4. Walter, D.O., Rhodes, J.M., Adey, W.R.: Discriminating among states of consciousness by EEG measurements: a study of four subjects. Electroencephalography and Clinical Neurophysiology 22, 22-29 (1967)

5. Walter, D.O., Rhodes, J.M., Kado, R.T., Adey, W.R.: A normative library of the human EEG, assessed by computer analysis in relation to behavioral states. Electroencephalography and Clinical Neurophysiology 23, 87-88 (1967)

6. Berka, C., Davis, G., Johnson, R.J., et al.: Psychophysiological Profiles of Sleep Deprivation and Stress during Marine Corps Training. Sleep 30, A132 (2007)

7. Bodenstein, G., et al.: Computerized EEG pattern classification by adaptive segmentation and probability-density-function classification. Description of the method. Computers in Biology and Medicine 15, 297-313 (1985)

8. Creutzfeldt, O.D., Bodenstein, G., Barlow, J.S.: Computerized EEG pattern classification by adaptive segmentation and probability density function classification. Clinical evaluation. Electroencephalography and Clinical Neurophysiology 60, 373-393 (1985)

9. Kramer, A., Parasuraman, R.: Neuroergonomics: Application of Neuroscience to Human Factors. In: Caccioppo, J.T., Tassinary, L.G., Berntson, G.G. (eds.) Handbook of Psychophysiology. Cambridge University Press, New York (2005)

10. Parasuraman, R.: Neuroergonomics: Research and Practice. Theoretical Issues in Ergonomics Science 4, 5-20 (2003)

11. Parasuraman, R.: Neuroergonomics: The Brain at Work. Oxford University Press, New York (2005)

12. Peltoranta, M., Pfurtscheller, G.: Neural network based classification of non-averaged event-related EEG responses. Medical and Biological Engineering and Computing 32, 189-196 (1994)

13. Pojman, N., Johnson, R.J., Kintz, N., et al.: Assessing fatigue using EEG classification metrics during neurocognitive testing. In: 23rd Annual Meeting of the Associated Professional Sleep Societies, LLC, Seattle, WA (Submitted, 2009) 
14. Wilson, G., Fisher, F.: Cognitive task classification based upon topographic EEG data. Biological Psychology 40, 239-250 (1995)

15. Berka, C., Johnson, R., Whitmoyer, M., et al.: Biomarkers for Effects of Fatigue and Stress on Performance: EEG, P300 and Heart Rate Variability. Human Factors and Ergonomics Society, New York (2008)

16. Berka, C., Levendowski, D., Cvetinovic, M., et al.: Real-time Analysis of EEG Indices of Alertness, Cognition, and Memory Acquired with a Wireless EEG Headset. International Journal of Human-Computer Interaction 17, 151-170 (2004)

17. Berka, C., Levendowski, D., Davis, G., et al.: Nicotine Administration and Withdrawal Effects on EEG metrics of Attention, Memory and Workload: Implications for Cognitive Resource Allocation. In: Schmorrow, D., Stanney, K., Reeves, L. (eds.) Augmented Cognition: Past, Present and Future, Foundations of Augmented Cognition, pp. 174-183. Strategic Analysis, Inc., Arlington (2006)

18. Berka, C., Levendowski, D., Konstantinovic, Z.R., et al.: Detection of drowsiness with realtime analysis of the electroencephalogram (EEG). In: National Institutes of Health SBIR Conference, Bethesda, MD (2000)

19. Berka, C., Levendowski, D., Ramsey, C.K., et al.: Evaluation of an EEG-Workload Model in an Aegis Simulation Environment. In: Caldwell, J.A., Wesensten, N.J. (eds.) Proceedings of SPIE Defense and Security Symposium, Biomonitoring for Physiological and Cognitive Performance during Military Operations, vol. 5797, pp. 90-99. SPIE: The International Society for Optical Engineering, Orlando, FL (2005)

20. Berka, C., Levendowski, D., Westbrook, P., Davis, G., Lumicao, M., Olmstead, R., Popovic, M.V., Zivkovic, V., Ramsey, C.: EEG Quantification of Alertness: Methods for Early Identification of Individuals Most Susceptible to Sleep Deprivation. In: Caldwell, J.A., Wesensten, N.J. (eds.) Proceedings of SPIE Defense and Security Symposium, Biomonitoring for Physiological and Cognitive Performance during Military Operations, vol. 5797, pp. 78-89. SPIE: The International Society for Optical Engineering, Orlando, FL (2005b)

21. Berka, C., Levendowski, D.J., Konstantinovic, Z.R., et al.: Detection of electroencephalographic indices of drowsiness in realtime using a multi-level discriminant function analysis. Sleep 23, A243-A244 (2000)

22. Berka, C., Levendowski, D.J., Lumicao, M.N., et al.: EEG correlates of task engagement and mental workload in vigilance, learning, and memory tasks. Aviation, Space and Environmental Medicine 78, B231-B244 (2007)

23. Mitler, M.M., Westbrook, P., Levendowski, D.J., et al.: Validation of automated EEG quantification of alertness: methods for early identification of individuals most susceptible to sleep deprivation. Sleep 25, A147-A148 (2002)

24. Westbrook, P., Berka, C., Levendowski, D., et al.: Quantification of Alertness, Memory and Neurophysiological Changes in Sleep Apnea Patients Following Treatment with nCPAP. Sleep 27, A223 (2004)

25. Westbrook, P., Berka, C., Levendowski, D.J., et al.: Biobehavioral quantification of alertness and memory in patients with sleep apnea. Sleep 25, A49-A50 (2002)

26. Craven, P.L., Belov, N., Tremoulet, P., et al.: Cognitive Workload Gauge Development: Comparison of Real-time Classification Methods. In: Schmorrow, D., Stanney, K., Reeves, L. (eds.) Augmented Cognition: Past, Present and Future, Foundations of Augmented Cognition, pp. 66-74. Strategic Analysis, Inc., Arlington (2006)

27. Poythress, M., Russell, C., Siegel, S., et al.: Correlation between Expected Workload and EEG Indices of Cognitive Workload and Task Engagement. In: Augmented Cognition 2006 Conference (2006) 
28. Poythress, M., Russell, C., Siegel, S., et al.: Correlation between Expected Workload and EEG Indices of Cognitive Workload and Task Engagement. In: Schmorrow, D., Stanney, K., Reeves, L. (eds.) Augmented Cognition: Past, Present and Future, Foundations of Augmented Cognition, pp. 32-44. Strategic Analysis, Inc., Arlington (2006)

29. Stevens, R., Galloway, T., Berka, C.: Allocation of Time, EEG-Engagement and EEGWorkload Resources as Scientific Problem Solving Skills are Acquired in the Classroom. In: Augmented Cognition 2007 Conference (2007)

30. Stevens, R., Galloway, T., Berka, C.: EEG-Related Changes in Cognitive Workload, Engagement and Distraction as Students Acquire Problem Solving Skills. In: The User Modeling Conference, Athens, Greece (2007)

31. Dickson, B.T.: The Cognitive Cockpit - a test-bed for Augmented Cognition. In: 1st International Conference on Augmented Cognition, Las Vegas, NV (2005)

32. Kinces, W.E.: DaimlerChrysler's closed loop integrated prototype: Current status and outlook. In: 1st Augmented Cognition International, Las Vegas, NV (2005)

33. Ververs, P.M., Whitlow, S., Dorneich, M., Mathan, S.: Building Honeywell's Adaptive System for the Augmented Cognition Program. In: 1st International Conference on Augmented Cognition, Las Vegas, NV (2005)

34. Mathan, S., Whitlow, S., Dorneich, M., Ververs, P., Davis, G.: Neurophysiological Estimation of Interruptibility: Demonstrating Feasibility in a Field Context. In: Augmented Cognition 2007 Conference (2007)

35. Berger, H.: Uber das Elektroenzephalorgam des Menschen. Arch Psychiatr. Nervenk 90, 527-570 (1929) 